Referencia para citar este artículo: Hedrera-Manara, L., \& Álvarez-Martínez-Conde, C. (2018). Los niños y las niñas recuerdan: memoria colectiva y participación en Memorial Paine, Chile. Revista Latinoamericana de Ciencias Sociales, Niñez y Juventud, 16(2), 961-977. doi:https://doi.org/10.11600/1692715x.16221

\title{
Los niños y las niñas recuerdan: memoria colectiva y participación en Memorial Paine, Chile*
}

\author{
LUCIANA HEDRERA-MANARA ** \\ Psicóloga Universidad de Chile, Chile. \\ CATALina ÁlVAREZ-MaRTÍNEZ-CONDE*** \\ Psicóloga Universidad de Chile, Chile.
}

Artículo recibido en mayo 2 de 2017; artículo aceptado en agosto 4 de 2017 (Eds.)

- Resumen (analítico): en este artículo reflexionamos sobre la relación entre memoria colectiva, infancia y participación, a partir de una intervención pedagógica organizada en un memorial de Santiago de Chile. Utilizamos una metodología cualitativa, teniendo como principales resultados que las memorias de las niñas y los niños se producen en una relación de co-construcción con los sujetos adultos, atravesadas por relaciones de poder que permiten distintas dinámicas de participación. Estos vínculos pueden reproducir memorias centradas en la víctima, en lo familiar y en el duelo, así como memorias transformadoras de la resistencia y la lucha social, en las cuales cobra importancia la apropiación e identificación de las nuevas generaciones con el lugar de memoria. Concluimos con una reflexión sobre la agencia de las niñas y los niños en el campo de la memoria y los derechos humanos.

Palabras clave: Memoria colectiva, infancia, dictadura, participación social, Chile (Tesauro de Ciencias Sociales de la Unesco).

\section{Children remember: Collective memory and participation at Memorial Paine, Chile}

- Abstract (analytical): The purpose of this article is to reflect on the relationship between collective memory, childhood and participation in a pedagogical intervention that occurred in a memorial in Santiago, Chile. The authors used an ethnographical qualitative methodology, with the results showing that children's memories are developed in co-construction with adults, a process influenced by power relations between participants and that enable different dynamics of participation. These links may reproduce memories that are focused on the victim, their family circles and mourning, as well as transformative memories of resistance and social struggle in which the appropriation and identification of children with the memorial become important. The authors conclude by reflecting on the agency of children in the fields of memory and human rights.

\footnotetext{
* Este artículo de investigación científica y tecnológica (Otras ciencias sociales: área interdisciplinaria), se basa en la memoria de título de psicología «Los niños también recuerdan: Memoria, Infancia y Participación en Memorial Paine, Un Lugar para la Memoria». Este estudio lo elaboramos en el marco del Proyecto Fondecyt Regular $N^{\circ} 1140809$ «Memorias de la Violencia Política en Chile: Narrativas Generacionales del período 1973-2013». Financiado por Conicyt Chile (Comisión Nacional de Investigación Científica y Tecnológica), y patrocinado por el Programa de Psicología Social de la Memoria de la Universidad de Chile. La investigación fue iniciada el 13 de marzo de 2014 y finalizada el 25 octubre de 2015.

** Psicóloga de la Universidad de Chile. Miembro del Programa Psicología Social de la Memoria, Universidad de Chile. Orcid: 0000-0003-2975-727X. Índice H5: 1. Correo electrónico: lu.hedrera@gmail.com

*** Psicóloga de la Universidad de Chile. Miembro del Programa Psicología Social de la Memoria. Universidad de Chile. Orcid: 0000-0002-2137-965X. Índice H5: 1. Correo electrónico: cata.alvarezmc@gmail.com
} 
Key words: Collective memory, childhood, dictatorship, social participation, Chile (Unesco Social Science Thesaurus).

\section{As crianças lembram: Memória coletiva e participação no Memorial Paine, Chile}

- Resumo (analítico): Neste artigo reflexionamos a relação entre lembrança coletiva, infancia e participação, a partir de uma intervenção pedagógica organizada em um memorial da regiao metropolitana do Chile. Utilizamos o método qualitativo do tipo etnográfico, tendo como principais resultados que as lembranças das crianças se produzem numa relação conjunta com os adultos, cruzadas por relações de poder que permitem diferentes dinâmicas de participação. Estes vínculos podem reproduzir lembranças centradas na vítima, na familia e no luto, assim como lembranças transformadoras centradas na resistencia e na luta social, nas quais cobra importancia a apropriação e identificação das crianças, no lugar da lembrança. Concluimos com uma reflexão sobre a ação das crianças no campo da lembrança e dos direitos humanos.

Palavras-chave: Memoria coletiva, infancia, ditadura, participação social, Chile (Tesauro de Ciências Sociais da Unesco).

\section{-1. Introducción. -2. Antecedentes: el memorial de Paine -3. Herramientas teóricas: infancia y memoria colectiva -4. Metodología. -5. Resultados. -6. Conclusiones. -Lista de referencias.}

\section{Introducción}

En Chile, con el retorno a la democracia luego de 17 años de dictadura cívico-militar, se ha dado paso a una cultura de la memoria (Reyes, Muñoz, \& Vázquez, 2013), que entre sus principios abarca la necesidad de transmitir lo ocurrido en el pasado reciente por medio de distintas iniciativas, destacando entre ellas la construcción de diversos lugares de memoria en el ámbito público ${ }^{1}$. El Estado y las distintas organizaciones de derechos humanos y de la sociedad civil, han manifestado la intención de incorporar a las nuevas generaciones en la discusión sobre memoria colectiva, derechos humanos y democracia (Corporación Opción, 2013; Veneros \& Toledo, 2009), entendiendo particularmente a los niños, niñas y adolescentes como actores sociales clave en la transformación de la realidad pasada y presente de la comunidad².

La visibilización de las nuevas generaciones en la construcción de memoria, nos hace preguntarnos por la manera en que los niños y las niñas son entendidos en estos procesos, por el rol de su participación y por los efectos que esto tiene en las memorias que son construidas. En este artículo buscamos reflexionar sobre estas cuestiones a partir de una investigación sobre las memorias que niños y niñas construían en una intervención pedagógica desarrollada en Memorial Paine, Un Lugar para la Memoria (en adelante, Memorial Paine) durante el año 2014.

En general, en el imaginario colectivo y en el ámbito de los derechos humanos, la construcción de memoria de las nuevas generaciones es entendida dentro de los marcos intergeneracionales de la transmisión del recuerdo, considerando a los niños y niñas como una suerte de guardianes de la

1 Desde el año 1990 con el retorno a la democracia y hasta la actualidad, se han construido más de 230 lugares de memoria en todo Chile (Piper, Reyes, Fernández, Hevia, Badilla, \& Olivari, 2011), siendo impulsada su construcción y gestión principalmente desde organizaciones de derechos humanos y agrupaciones de familiares de las víctimas de la dictadura, y en menor medida desde el ámbito estatal. Estos tienen como objetivo mantener patente el recuerdo por medio del mantenimiento de estos lugares y de la realización de diversas actividades y eventos destinados a generar una cultura de promoción de los derechos humanos (Programa de Derechos Humanos, 2013).

2 Esta es una necesidad que ha emergido en el curso de la última década, acompañada del desarrollo de políticas de reparación simbólica preocupadas por el tratamiento de la memoria colectiva y por el reconocimiento de las memorias de las víctimas a nivel social. En ese sentido, la preocupación por la integración de las nuevas generaciones en la construcción de memoria, se ha dado principalmente a través de la implementación de políticas educativas con las que se busca establecer contenidos mínimos en el currículum escolar, los cuales quedan sin embargo al arbitrio de los establecimientos educacionales respecto de qué y cómo abordar dichos contenidos (Delgadillo, 2016). Bajo este contexto, los lugares de memoria han desarrollado distintas actividades pedagógicas que buscan ser un insumo para los establecimientos que desean enseñar sobre el período de dictadura y sobre todo lo que aquello significa (Salomone \& Cea, 2013). 
memoria de las generaciones anteriores (Habashi, 2013). Así, esta trasmisión es comprendida como un legado que la generación protagonista deja a la generación sucesora, designando la memoria como propiedad de unos que después es transferida a los demás (Cruz, Reyes, \& Cornejo, 2012). Esto si bien ha permitido posicionar a los niños y niñas como sujetos de memoria colectiva, a nuestro entender lo ha hecho desde una visión adultocéntrica y tradicional, restringiendo su participación en ello.

Por su parte, en las ciencias sociales la infancia ha tenido un espacio secundario, ya que no se ha considerado a niños y niñas como sujetos significativos en los procesos de desarrollo y transformación social (Vergara, Peña, Chávez, \& Vergara, 2015), al verlos bajo una mirada idílica y romántica como personas inocentes y desconocedoras del mundo, lo que ha llevado a supeditar la vida del niño o niña a la del individuo adulto. Este panorama no es ajeno al de la memoria, ya que de la misma manera, en los procesos de transmisión intergeneracional, se entiende que los niños y niñas también dependen y necesitan de las personas adultas para recordar, subordinando así sus memorias a las experiencias de otros individuos en un rol pasivo (Habashi, 2013).

Para problematizar estas visiones desde la agencia de los niños y niñas, tomamos perspectivas provenientes de la psicología social crítica y de la memoria (Íñiguez, 2005; Piper, 2002; Piper, Fernández, \& Iñíguez, 2013), del Feminismo y los Nuevos Estudios Sociales de la Infancia (Burman, 2007, 2013; Castillo-Gallardo \& González-Celis, 2015; James \& Prout, 2003; Mannion, 2007; Vergara, Chávez, Peña, \& Vergara, 2016). De ellas nos valimos para relevar la agencia de la infancia en los procesos sociales y políticos de los que forman parte, considerando a los niños y niñas, en el análisis de esta experiencia particular de construcción de memoria, como actores sociales relevantes que aportan al complejo campo de la memoria colectiva, de los derechos humanos y de la democracia.

Reflexionar sobre estas cuestiones permite aportar con visiones agenciales sobre la niñez, así como propiciar la generación de espacios de transformación, toda vez que nuevos sujetos y significados son incluidos en el tejido social de la memoria.

\section{Antecedentes: Memorial Paine}

El Memorial Paine es un espacio al aire libre en el que se recuerda a las víctimas locales de la dictadura cívico-militar chilena. Paine es una localidad rural al sur de la Región Metropolitana de Santiago, caracterizada tristemente por ser una de las comunas con la mayor cantidad de víctimas de violencia política en relación con la densidad de su población (Comisión Nacional de Reparación y Reconciliación, 1996) ${ }^{3}$. El fuerte impacto de la violencia política tuvo como consecuencias la desarticulación de las relaciones sociales de la comunidad, la descomposición de familias debido a la represión, y una marcada polarización social que continúa hasta el día de hoy (Maureira, 2008). En este contexto, la Agrupación de Familiares de Detenidos Desaparecidos y Ejecutados de Paine tuvo una acción fundamental respecto de la búsqueda de verdad y justicia, enarbolando — una vez finalizada la dictadura - una serie de iniciativas en el marco de la memoria y los derechos humanos.

Una de ellas fue la construcción colectiva del Memorial Paine, inaugurado en el año 2008 y compuesto por mosaicos que recuerdan a las personas de la comuna detenidas, desaparecidas y ejecutadas durante la dictadura: hombres que participaron en organizaciones políticas y laborales que tuvieron un rol fundamental en el establecimiento de la Reforma Agraria durante la década de 1960 y principios de 1970. Cada mosaico recuerda la historia personal y familiar de las víctimas, y en su conjunto narran la historia rural y colectiva de la localidad.

Asimismo, cuenta con postes de madera que se yerguen entre los mosaicos, y que representan el horizonte geográfico y a la comunidad de Paine (Imagen 1). Otro elemento llamativo en el lugar son las fotografías de cada una de las víctimas exhibidas en la oficina del Memorial Paine (Imagen 2).

3 Según el Informe Rettig (Comisión Nacional de Reparación y Reconciliación, 1996), en Paine se registran 70 personas detenidas, desaparecidas y ejecutadas por motivos políticos durante la dictadura, siendo en la actualidad más de mil los descendientes directos de las víctimas (Hite, 2013), compuestos por la segunda y tercera generación, además de una red de amigos/as, conocidos y amigas, parientes indirectos y otros. 
Imagen 1. Postes y Mosaicos del Memorial Paine 4

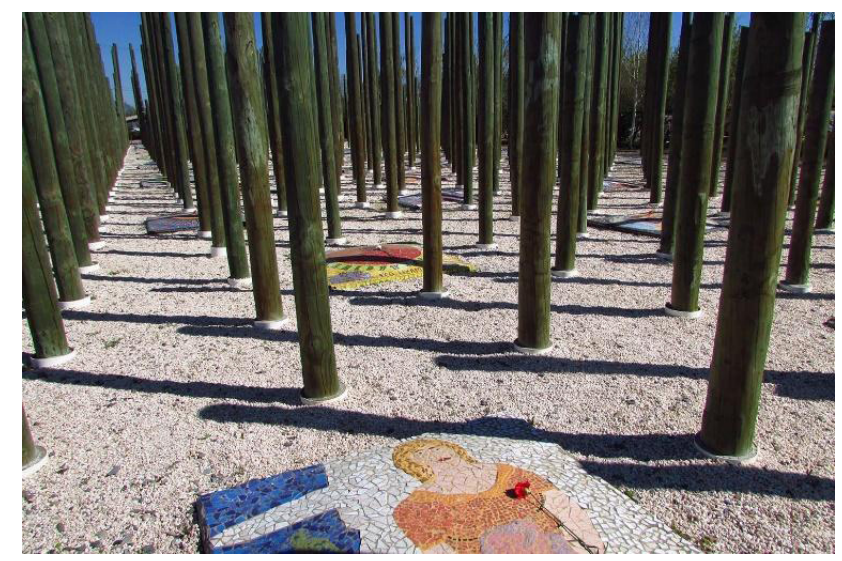

Imagen 2. Fotografias de las personas recordadas en Memorial Paine

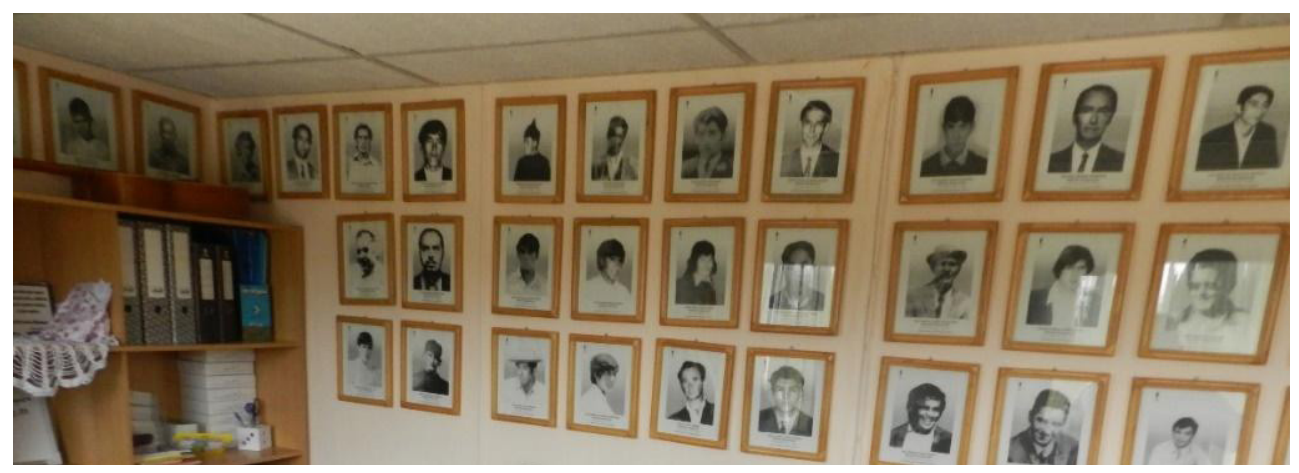

En este espacio se realizan distintas actividades diseñadas y ejecutadas por el equipo de educación y visitas guiadas del memorial. una de ellas es la realización de encuentros educativos con niños, niñas y adolescentes provenientes de escuelas y organizaciones de Paine, que surgió de la intención del Memorial por posicionarse como espacio relevante para la comunidad, atendiendo el problema de la fragmentación social y la existencia de memorias contrapuestas y conflictivas en Paine (Memorial Paine, 2013). Los encuentros corresponden a una actividad pedagógica orientada a reflexionar sobre derechos humanos, memoria colectiva y democracia, por medio de la transmisión de memoria sobre lo ocurrido durante la dictadura, ya que se considera que por medio del conocimiento de esta historia los niños y niñas pueden contribuir a cambiar las visiones que existen en su realidad social (Memorial Paine, 2013).

Entre los contenidos que se abordan está la transmisión de lo ocurrido en Paine durante la Reforma Agraria, la Dictadura Militar y la acción de lucha de la Agrupación de Familiares, culminando con una reflexión sobre la importancia del Memorial y el ejercicio de memoria. Las dinámicas más relevantes de los encuentros son la exposición de un relato sobre la historia local y una actividad de interpretación libre de mosaicos, todas acompañadas por los monitores y monitoras del equipo. En estas actividades se despliegan prácticas de memoria particulares, en las cuales se configuran y reconfiguran sentidos del pasado y del presente a través de interacciones, prácticas y discursos. Los significados y relaciones que en ellas se gestan son los que nos interesa analizar en este artículo, en tanto son una fuente de información primordial para entender el rol de las nuevas generaciones en la construcción de la memoria colectiva y de la realidad política y social de nuestras sociedades.

$4 \quad$ Todas las fotografías son fuentes de elaboración propia. 


\section{Infancia y memoria colectiva}

\subsection{El deber de la transmisión}

En el ámbito de la memoria colectiva, el rol de los niños y niñas ha sido entendido principalmente desde las teorías de la transmisión intergeneracional del recuerdo (Veneros \& Toledo, 2009), teorías que en sus bases comparten visiones más bien tradicionales de la infancia. Desde estas perspectivas, la transmisión intergeneracional es definida como la reconstrucción del pasado por medio de conocimientos culturales compartidos por generaciones anteriores (Jelin, 2002; Kaufman, 2006), que tendrían como resultado una posmemoria o «la memoria de la generación siguiente a la que padeció o protagonizó los acontecimientos» que se recuerdan (Sarlo, 2005, p. 126). Así, la posmemoria es entendida como esencialmente distinta a la memoria, basando dicha diferencia en la presencia o ausencia de una experiencia directa por parte de las generaciones con el período recordado.

De este planteamiento también se desprende que la construcción de memoria de los niños y niñas está ligada a lo que los individuos adultos y sus instituciones les transmiten, siendo la transmisión intergeneracional considerada un deber relacionado con la mantención de una historia común particular (Veneros \& Toledo, 2009). Se entiende además que la transmisión intergeneracional y, por ende, la posmemoria, se caracteriza por su capacidad sanadora, pues se asume que las generaciones que vivieron los hechos recordados están dañadas, y que por consecuencia deben transmitir estas memorias a las nuevas generaciones para que transformen la forma como se concibe lo ocurrido en el pasado (Salomone \& Cea, 2013; Sarlo, 2005).

Las principales instituciones por medio de las cuales se produce la transmisión son la familia y la escuela (Bustelo, 2005; Leccardi \& Feixa, 2011), desde donde las nuevas generaciones obtienen información para significar el pasado. Estos espacios, a pesar de que la mayoría de las veces entregan información de manera unilateral, se conforman en puntos de conflicto en los que niños y niñas pujan por construir sus propias versiones (Corporación Opción, 2013). Sin embargo, tras la labor de estas instituciones se encuentra el supuesto de que la niñez no es artífice de su mundo sociocultural, por lo que la relación entre el sujeto y su cultura —y por ende con su memoria - sería de carácter transitivo y no reflexivo (Batallán \& Campanini, 2008).

Para nuestro propósito, buscamos un distanciamiento de las teorías tradicionales de la transmisión intergeneracional, ya que si bien plantean visiones interesantes de la construcción de memoria de las nuevas generaciones, como su capacidad transformadora, nos parece que obvian $-\mathrm{y}$ a la vez naturalizan - las maneras en que se valida lo que se transmite y desde quiénes o a quiénes lo transmiten, coartando las posibilidades de acción en tanto determinan de antemano los lugares que los sujetos ocuparán en estos procesos.

Basar las distinciones que hacemos entre sujetos a partir de sus experiencias es algo peligroso ya que, como dice Joan Scott (2001), concebir la experiencia como algo que un sujeto tiene, evita el análisis de esas categorías como producciones históricas y relacionales.

\subsection{La co-construcción de la memoria colectiva}

Durante los últimos años, en las ciencias sociales las perspectivas feministas han llamado a comprender desde una mirada crítica las temáticas sobre infancia, desafiando la manera clásica de entender el mundo infantil (Burman \& Stacey, 2010). Frente a una epistemología moderna que entiende a los sujetos como categorías determinadas e inamovibles, perspectivas críticas han planteado considerar en principio, y fundamentalmente, a los niños y niñas como sujetos social e históricamente construidos (Burman, 2008, 2013; Vergara et al., 2016). Concebir la infancia de esta forma permite entender que se encuentra constituida por variables culturales y de género, con opiniones, necesidades y demandas que no están necesariamente supeditadas a las personas adultas, a la familia y a las instituciones de protección. Por esto, desde visiones socio-construccionistas de la 
memoria (Habashi, 2013; Piper, Montenegro, Fernández, \& Sepúlveda, 2017; Reyes, 2009), se plantea la necesidad de pensar en los niños y niñas como co-constructores activos de la memoria colectiva, de la identidad nacional y de la realidad social, entendiendo su accionar en dicha construcción no solo como transformadores de las relaciones sociales - lo que nos hablaría de una perpetuación de la mirada romántica e idealizada-, sino también como productores y reproductores de los sistemas sociales en los que vivimos (Vergara et al., 2015).

Dicho esto, entenderemos la memoria como acción social, política y cultural que genera significados e interpretaciones del pasado hechas desde el presente (Vázquez, 2001). En ese sentido, las narraciones de memoria implican que el pasado puede ser interpretado de diversas maneras, siendo una construcción social que contribuye a elaborar la realidad en la que la sociedad y los individuos se reconocen. Debido a su carácter relacional, esta definición nos invita a pensar que la acción de los niños y niñas no puede ser entendida como separada de la acción de los demás agentes que participan en la construcción de memoria.

Asimismo, la memoria se realiza tanto lingüística como performativamente, a través de prácticas de recuerdo reiteradas y constreñidas a ciertas normas, por medio de las que se construyen identidades particulares. Es en el discurso y en las prácticas de memoria donde los sujetos se conforman como tales (Piper et al., 2013). Por tanto, no es que los niños y niñas tengan una memoria esencialmente distinta de la de otros actores, sino que son justamente sus prácticas y discursos sobre el pasado lo que las constituye como niños y niñas que recuerdan.

\subsection{Lugares de memoria y participación social}

Las acciones y los contextos en que los niños y las niñas actúan han sido pensados como distintos a los de las personas adultas, anclándolos a ámbitos familiares, despolitizados y privados, con poca incidencia en el ámbito público (Vergara, 2009). La memoria, como acción social y política, nos permite pensar en la participación de las distintas generaciones y especialmente a los y niñas en los múltiples ámbitos de acción política que no necesariamente pertenezcan a la escuela y a la familia. Este carácter político y social de la memoria se despliega en variados contextos de relación, en los cuales existen sujetos con distintos grados de legitimidad que buscan intervenir en los sistemas de significación del presente (Reyes et al., 2013). Así, y como señalan Piper et al. (2017):

La memoria se constituye como un centro articulador de lo social a través de la construcción del pasado, la explicación del presente, y como sostén identitario y eje articulador de prácticas políticas, a partir de las relaciones de poder y resistencia en el presente ${ }^{5}$. (p. 4)

El ámbito público de la memoria, considerado desde estas dimensiones, sería un espacio marcado por su carácter conflictivo, por lo que es primordial pensar que los niños y niñas, cuando participan en la construcción de memoria, buscan legitimar sus decisiones e interacciones a partir de valores y desde el compromiso afectivo con los demás, en una red marcada por las diferencias de poder (Vergara et al., 2015). Estas disputas producen distintos espacios de diálogo, entre otros los lugares de memoria, los cuales entendemos como espacios significativos que son usados y apropiados a través de prácticas de recuerdo que interpretan sentidos del pasado (Piper \& Hevia, 2012). La elaboración de los significados y el tipo de relaciones que los sustentan respecto al espacio, determinan de qué manera las personas se vinculan con el Memorial y cómo se apropian de el (Álvarez-MartínezConde \& Hedrera, 2013), jugando un rol importante el establecimiento de vínculos afectivos con el espacio. Esto se produce por medio de la identificación y de la posibilidad de acción/transformación que los sujetos tengan en el lugar, entendiendo que su participación en la construcción del espacio simbólico es fundamental para que ellos puedan pensarlo como un aporte para la comunidad (Viú \& Buzaglo, 2006). Por esto los memoriales, además de relatar una situación histórico-política, también contribuyen a la creación y transformación dinámica de la memoria colectiva (Piper et al., 2017).

$5 \quad$ La traducción fue realizada por los autores del artículo. 
También entendemos este lugar como un espacio intergeneracional, donde se despliegan performances relacionales de infancia y adultez que atraviesan la construcción de memoria, produciendo simultáneamente posicionamientos imbuidos en relaciones de poder cambiantes (Mannion, 2007). Al respecto, es atingente el concepto de dialogía intergeneracional (Reyes, 2009; Reyes, Cornejo, Cruz, Carrillo, \& Caviedes, 2014), el cual refiere al espacio relacional que se construye entre distintas generaciones al hacer memoria del pasado, entendiendo que los discursos se encuentran atravesados por los tipos de vínculos entre las posiciones generacionales, lo que se traduce en relaciones de apoyo, de conflicto, de diálogo, entre otros.

Tener estas visiones implica centrarse tanto en los niños y niñas como en la relación entre niños, niñas y sujetos adultos, y en la manera en que este vínculo genera prácticas en determinados espacios, con el fin de promover mayor horizontalidad. En ese sentido, entenderemos la participación como un proceso relacional, el cual determina el involucramiento de las personas en una relación de reflexión y acción social entre agentes que ocupan posiciones de poder diversas, con variados grados de legitimidad para opinar, decidir y actuar (Montenegro, 2001). Las acciones y discursos que los niños y niñas realizan al habitar un Memorial e interpretar el pasado, también contribuyen a una forma de participación social y política relevante. La atención a estos procesos es lo que nos permite complejizar la comprensión sobre cómo las memorias de la infancia son construidas, ya que es justamente en el nivel de su participación donde vemos el carácter de las relaciones que se dan entre los sujetos que allí intervienen.

\section{Metodología}

Basamos la investigación en una metodología cualitativa utilizando primordialmente la observación participante y entrevistas etnográficas, para acercarnos e interpretar las prácticas e interacciones que realizaron niños, niñas y personas adultas en el Memorial. Esto nos permitió comprender los procesos dentro de su contexto, considerando las características y experiencias personales de los actores en una determinada situación (Guber, 2011; Restrepo, 2015). Asimismo, adoptamos una noción situada del conocimiento (Haraway, 1995), en tanto asumimos que cualquier conocimiento responde a posiciones parciales, lo que nos aleja de una intención totalitaria del mismo y nos permite responsabilizarnos por la mirada particular de la realidad social que entregamos, así como por la producción misma de dicha mirada.

De este modo, siguiendo a Cruz et al. (2012), consideramos que en el campo de la investigación social cualitativa operan premisas del conocimiento situado en tanto el sujeto investigador se afecta y es parte constitutiva de aquello que se investiga. En este proceso, quienes investigamos tenemos preocupaciones metodológicas, pero también inquietudes epistémico-políticas sobre cómo llevamos a cabo estas prácticas de producción de conocimiento.

Para este estudio, la entrada al campo estuvo dada por la posición de interventoras al ser parte del equipo de educación del Memorial Paine, por lo que asumimos al mismo tiempo dicho rol y el de investigadoras, lo cual significó mantener una actitud reflexiva y atender a las posiciones que íbamos tomando en el desarrollo del estudio. De esta manera, investigación y práctica se dieron como procesos interrelacionados y dependientes, incorporando de forma constante las reflexiones y problematizaciones a medida que producíamos los datos y trabajábamos sobre ellos. La multiplicidad de roles implicó la asunción de relaciones más directivas con los niños y niñas dadas por el rol de interventoras, y a su vez, vínculos más horizontales referidos a las intenciones investigativas, donde buscábamos tensionar la relación sujeto adulto-niño/niña. Considerando que nuestro estudio implicó un trabajo con infancia, creemos que esta variable es fundamental, ya que las investigaciones en este ámbito tienen un carácter particular por la imbricación de las relaciones de poder entre investigadorainvestigada y adulta-niña (Haudrup, 2004). Para tensionar estas diferencias, realizamos lo que Pia 
Haudrup (2004) llama el acto de observar y de escuchar atentamente, donde lo primero implica que antes de iniciar cualquier involucramiento en una práctica desarrollada por niños y niñas, es necesario observar y entender lo que se realiza, con el fin de entrar en sintonía con la dinámica; mientras que lo segundo significa escuchar hasta el final, sin interrumpir su discurso, dejando que niños y niñas decidan cuándo pueden terminar su intervención.

Concretamente, observamos y participamos de seis encuentros en el Memorial Paine, los cuales se llevaron a cabo entre los meses de mayo y agosto del año 2014, en los que participaron grupos de 20 a 30 niños y niñas, entre los 8 y los 15 años de edad, provenientes de escuelas del sector, un profesor o profesora, y los monitores y monitoras del Memorial. En todos los encuentros hubo algún niño o niña familiar directo de las víctimas recordadas en el Memorial.

Hicimos el análisis de los datos en dos etapas consecutivas. En la primera, las observaciones fueron registradas en un diario de campo que generaron resultados descriptivos sobre las prácticas de todos los actores presentes, poniendo especial atención a los niños y las niñas ${ }^{6}$. También recurrimos a las guías con las que se desarrollaban las actividades, a las evaluaciones realizadas por el equipo de trabajo al final de la actividad, y a materiales documentales del Memorial sobre su historia y construcción. En la segunda etapa, construimos preliminarmente un relato descriptivo organizado en categorías con los datos producidos (Restrepo, 2015), y luego elaboramos un texto analítico donde articulamos los resultados, las interpretaciones y los elementos teóricos, para así comprender la construcción de memoria de los niños y las niñas junto a su participación.

\section{Resultados}

\subsection{La memoria como proceso relacional entre niños, niñas y adultos}

En el Memorial de Paine las acciones de los niños y las niñas se desarrollan con la compañía de personas adultas, por lo que la construcción de memoria está en estrecha relación con las formas en que ambos se relacionan.

Durante el encuentro, niños, niñas y sujetos adultos se van construyendo mutuamente en el espacio relacional, performando roles y posiciones desde donde actuar, los cuales se articulan en torno a responsabilidades distintas: los individuos adultos y la institución como encargados de la transmisión de memoria, y los niños y niñas como responsables de recibir dicha información, planteando posiciones de saber diferenciadas.

Siguiendo esta lógica de la transmisión intergeneracional del recuerdo se desplegaron vínculos jerárquicos entre las personas adultas y los niños y niñas. Esto se daba por medio del establecimiento de un guión previamente definido por el equipo para relatar lo ocurrido en la dictadura, privilegiando así su propia versión de la memoria. Como el guión estructuraba contenidos expositivos y directivos, los niños y niñas quedaban sujetos al aprendizaje de este relato, posicionando en primer lugar los intereses de la persona adulta, operando relaciones desiguales de poder-saber que ponían a los niños y niñas en un rol secundario de escucha y a las personas adultas como figuras de autoridad. Esto propiciaba actitudes de aburrimiento y desconcentración al no considerarse como posibilidad la intervención libre en el relato. Al respecto, un grupo de niñas criticó no haber podido conocer las historias de los mosaicos por brindar más tiempo a la exposición que hacían los monitores y monitoras, afirmando que esta se hizo muy extensa.

Un elemento que aportaba a estas dinámicas era la actualización del contexto escolar en el Memorial. Los niños y niñas asistían al Encuentro en horario de clases junto a su profesor o profesora.

$6 \quad$ Para ello utilizamos registros fotográficos y orales, los cuales fueron debidamente autorizados por el Equipo del Memorial Paine y por las escuelas participantes mediante el consentimiento de los padres, madres y directores responsables de los niños y niñas. Asimismo, a los niños y niñas participantes de los Encuentros se les informó sobre el estudio y se les consultó su intención de participar. 
A pesar de que este era un espacio distinto al aula, la figura de las personas adultas (monitores, monitoras y docentes) se actualizaba como figura de autoridad y control, restringiendo las acciones de los niños y niñas en tanto normaban sus intervenciones y recorridos en el espacio.

Esta forma de construcción de memoria se basa en el supuesto de que los niños y las niñas son desconocedoras de la realidad social y, por ende, únicamente receptoras de las memorias de otras personas, estando el sujeto adulto sindicado como el agente responsable de enseñar sobre el pasado. A su vez, los recuerdos previamente definidos como admisibles limitan los elementos que pueden aparecer en las narraciones de los niños y niñas, al generarse un marco normativo desde donde hacer memoria. En ese sentido, la infancia es entendida como relevante, pero en la medida en que se mantiene dentro de marcos de significación que permiten la emergencia de ciertas memorias y valores. Esto provoca la circunscripción de las acciones de los niños y niñas a ámbitos asociados al deber y a la validación de las memorias de otras generaciones. Dentro de este contexto, sus experiencias son secundarias, pues el aporte que pueden hacer a la construcción de memoria se producirá solo una vez que han «recibido» la memoria que los individuos adultos les entregan, dejando de ser entendidos estos procesos como co-relacionados e interdependientes (Habashi, 2013). Así, la niñez queda supeditada a los intereses del mundo adulto, lo que afecta el protagonismo que los niños y niñas podrían adquirir en la construcción de memoria, gracias al privilegio de relaciones de poder desiguales (Alfageme, Cantos, \& Martínez, 2003).

Es en estas relaciones donde se produce lo que Vázquez (2001) llama la domesticación del recuerdo, pues se fijan visiones hegemónicas que buscan privilegiar ciertos sentidos del pasado, invisibilizando y restringiendo la acción de los niños y las niñas al no propiciar el aporte de elementos novedosos. Asimismo, estas relaciones generan distanciamiento y dificultan el compromiso de los niños y niñas con las temáticas y la apropiación del espacio, ya que, por ejemplo, cuando interpretaban los mosaicos del Memorial y algunos monitores y monitoras les informaban de manera tajante los significados que las familias habían intentado plasmar en ellos, perdían el interés y limitaban su creatividad, quedándose con las interpretaciones oficiales.

Sin embargo, las relaciones marcadas por aspectos autoritarios podían ser tensionadas por vínculos más horizontales entre niños, niñas y sujetos adultos, los cuales privilegiaban en su despliegue el accionar de los niños y niñas como sujetos activos en la construcción de memoria, al potenciar los intereses y prácticas que a ellas y a ellos les hacían sentido respecto de su propia historia. Cuando los niños y niñas que tenían un vínculo familiar con alguna víctima del Memorial preguntaban a las monitoras y monitores por el estado actual de la búsqueda de sus parientes ${ }^{7}$, estos les informaban con datos e historias significativas, información que no habían podido obtener por otros medios.

Igualmente, mientras más se interpelaba a los niños y niñas con preguntas, o se los invitaba a reflexionar desde sus propias realidades, los diálogos y acciones se tornaron más dinámicas, ya que podían aportar elementos nuevos, construyendo un relato de manera conjunta con las y los adultos. En uno de los encuentros, asistió un niño que había participado en la construcción del mosaico de uno de sus familiares y mientras el monitor contaba los significados del mosaico, el niño agregó que el color verde representaba la pasión de su familiar por el fútbol, dato que luego fue incorporado al propio relato del Memorial. Otra actividad que permitía esta dinámica tuvo lugar antes de un Encuentro, donde el equipo del Memorial les pidió a los y las niñas que les preguntaran a sus familias cómo recordaban la vida antiguamente en el campo, antes de la dictadura. Esta actividad, al igual que la anterior, permitió una construcción de memoria de forma participativa, elaborando en el espacio un recuerdo compuesto por distintas fuentes, en las que los niños y niñas también podían aportar con información válida que otras personas desconocían, y construir sus propias memorias al respecto.

$7 \quad$ Es importante mencionar que la mayoría de las personas parientes de las víctimas desaparecidas o ejecutadas de Paine aún no han podido saber dónde o cuándo fueron asesinados sus familiares. En ese sentido, para los niños y niñas el Memorial también es un espacio que entrega valiosa información para ellas y sus familias. 
En ese sentido, las relaciones cooperativas permitían que el espacio se volviera significativo y abierto para conocer y compartir las memorias de los distintos actores, dando lugar a que los niños y niñas expresaran sus propias concepciones sobre el pasado, nutriendo con experiencias diversas el relato de memoria que se hacía durante el encuentro. Para ello, era importante que tanto niños y niñas como sujetos adultos presentes se encontraran abiertos al diálogo, desplazando la posición de saber autorizado hacia la construcción de un saber intergeneracional que comprende su riqueza en tanto responde a diversas fuentes de experiencia. Por su parte, la posición de agencia en la que se situaban los niños y niñas durante el ejercicio de memoria establecido por medio de estas relaciones cooperativas, propiciaba su compromiso con los propios objetivos del espacio, así como los del equipo a cargo. Luego de un encuentro particularmente participativo ${ }^{8}$, las niñas manifestaron su intención de difundir en su comunidad y familia lo que habían aprendido en el Memorial, con el fin de transformar la visión negativa que existía en Paine sobre el espacio9. De esta forma se puede ver que este tipo de relaciones desestabilizan las maneras normadas de hacer memoria, ya que los niños y niñas pueden desenvolverse como actrices relevantes al ser protagonistas de sus acciones y elaboraciones tanto sobre el pasado como sobre el presente.

\section{Imagen 3. Niñas y personas adultas conversando sobre un mosaico}

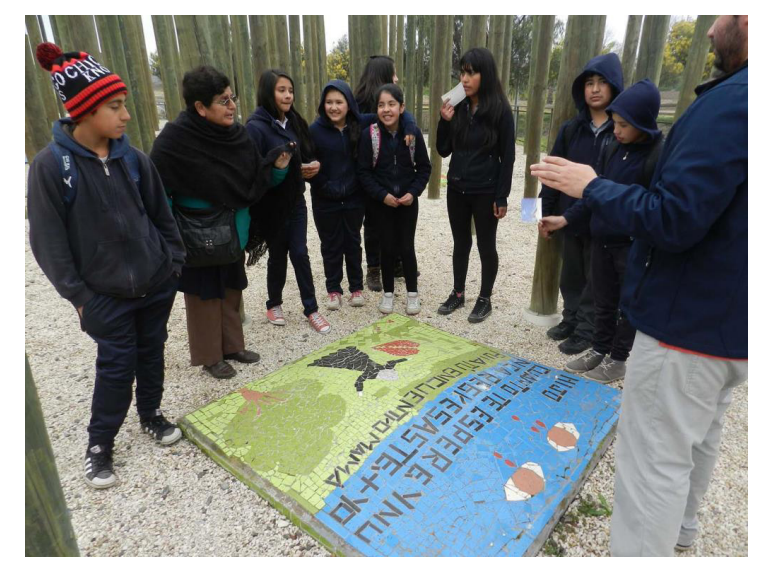

\subsection{La apropiación e identificación en el ejercicio de memoria}

Memorial Paine es un espacio caracterizado por estar al aire libre, con mosaicos de colores, postes y fotografías que llaman la atención de los niños y las niñas. En su uso se van posibilitando procesos de apropiación e identificación particulares entre los niños, las niñas y el espacio, los cuales tienen un rol fundamental en la construcción de memoria.

Cuando los niños y las niñas llegaban al Memorial, tenían el interés de descubrir el espacio, familiarizándose con el al correr, jugar, abrazar los mosaicos y postes, o al tomar fotografías. Estas acciones dan cuenta de maneras particulares de relacionarse con el lugar, dadas por el movimiento y el juego, en los que se van usando distintos rincones y periferias, movimientos discontinuos y zigzagueantes, lo que rompe con maneras pautadas y con normativas sobre la manera de recorrer el Memorial y hacer memoria, al plantear formas que les son propias. En este contexto, no hay sujeto

8 En este Encuentro la participación fue el norte de principio a fin; emergieron diálogos y debates en vez de exposición, y se dio mucho tiempo para la interpretación libre de mosaicos. Asimismo, los monitores y las monitoras tomaron un rol más de escucha, posibilitando que las niñas fueran más activas.

9 Debido a la participación de civiles en los crímenes y en la represión cometidos en Paine durante la dictadura, existe una fuerte polarización política en la comunidad, lo que ha hecho que algunos sectores - personas simpatizantes del régimen, victimarios y sus familiares, vecinos y vecinas atemorizadas - no se relacionen con el Memorial por no querer vincularse o por miedo a represalias, pero también por encontrar que es un espacio que incita al odio y a la división de la comunidad (Maillard \& Ochoa, 2014). 
que indique por dónde ir o que signifique los objetos más que ellas y ellos mismos, por lo que sus intereses están dados por todo aquello que el recorrido les provoca.

Imagen 4. Niñas explorando el Memorial

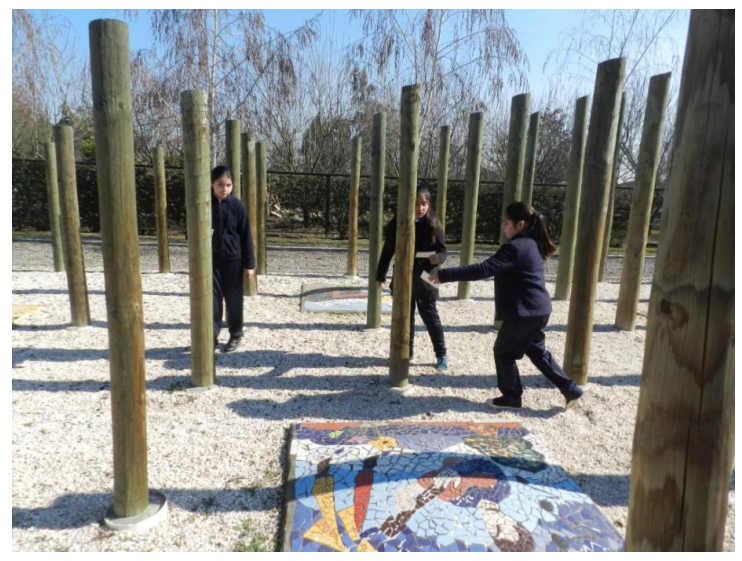

El lugar de memoria se configuraba como un soporte material de las experiencias compartidas por los distintos actores, posibilitando la apropiación del espacio. Los niños y las niñas en el lugar compartían sus historias familiares sobre el pasado, reconociéndose como parte de una historia común. La acción de compartir las memorias hace que los relatos personales y familiares se vuelvan públicos y disponibles para que otros sujetos se apropien de ellos. En los momentos de recorrido libre, los niños y las niñas familiares solían buscar las fotografías de sus parientes, haciendo parte de esta búsqueda a compañeros y compañeras que no tenían vínculo familiar — les pedían ayuda para encontrar el nombre de su pariente - ; en otros momentos, eran los niños y las niñas sin parentesco quienes iniciaban esas pesquisas motivadas por la curiosidad. Al encontrar las fotografías, los niños y las niñas implicadas en la búsqueda demostraban alegría y hacían comentarios sobre los parecidos físicos entre la persona encontrada y sus compañeros o compañeras, al tiempo que aparecían historias familiares relacionadas con la fotografía que se observaba. De esta forma, la búsqueda aportaba al establecimiento de vínculos afectivos y de identificación tanto a nivel familiar — por la validación de un parentesco en cuestión - y comunitario - por la resignificación de la fotografía, y por ende del espacio-, como parte de la historia comunitaria del grupo de niños y niñas gracias a su carácter más cercano.

En ese sentido, la apropiación de las memorias familiares, tanto propias como de otros, conforma en el espacio una comunidad de recuerdo que comparte temporalidades, espacios y pensamientos desde donde reconocerse (Halbwachs, 1968). Así, en las distintas narraciones y prácticas se elabora una memoria social alimentada por diversas experiencias, las cuales se legitiman al encontrar un lugar — social y material — en donde aparecer y ser reconocidas.

Como el Memorial contiene varios elementos de significación, tanto en el espacio como en el relato que se va haciendo se genera un contexto desde donde enunciar e interpretar el pasado. La dictadura, la represión, la desconfianza, la violencia, la lucha social y la resistencia se materializan en los objetos y se hacen carne en alusión a personas concretas que vivieron los hechos narrados por los niños y niñas. La interpelación que el lugar de memoria les hace a ellos y ellas por medio del testimonio físico, permite que el involucramiento en la construcción de memoria sea más efectivo, y por ende afectivo. Esto porque, por un lado, materializa aquellos discursos que impregnan nuestra sociedad en espacios, objetos y afectos, y por otro, porque impide la rigidización de la memoria, abriendo la posibilidad para la resignificación, generando comprensiones y sensaciones del pasado en el presente (Fernández, 2000; Piper \& Montenegro, 2016). 
En estos procesos, el Memorial interpela a los niños y las niñas desde distintos lugares, por ejemplo desde su historia familiar por medio de los mosaicos y las fotografías, y desde su comunidad a través de los postes de madera y de los aspectos rurales en los mosaicos. Así también, hay elementos en los mosaicos que refieren a experiencias de lucha política: banderas e insignias de colectivos político-campesinos, personas con los brazos alzados y el puño en alto, entre otros. Estas figuras propician una identificación desde la resistencia en la que los niños y niñas también pueden participar, construyendo una memoria que articula resistencias del pasado organizadas por otros sujetos y sus propias intenciones de cambio en el presente, haciendo que el espacio cobre sentido desde la recuperación y proyección de la acción colectiva. Lo dicho se puede apreciar en la Imagen 5, en la que se retrata el diálogo entre un grupo de niños y niñas y un monitor:

- Todos y todas: ¿Qué significa MIR?

- Monitor: MIR significa movimiento de izquierda revolucionaria; fue un colectivo político que estaba a favor de la revolución y la lucha armada. Fue uno de los más golpeados durante la dictadura.

- Juan: Eran jóvenes, se nota por las caras.

- Monitor: ¿Por qué les gustó?

- Diego: Por la historia.

- Romina: Se parece a nosotros, nosotros también queremos cambiar las cosas.

- Ignacio: Es distinto a los demás mosaicos.

Imagen 5. Interpretación de mosaico ${ }^{10}$

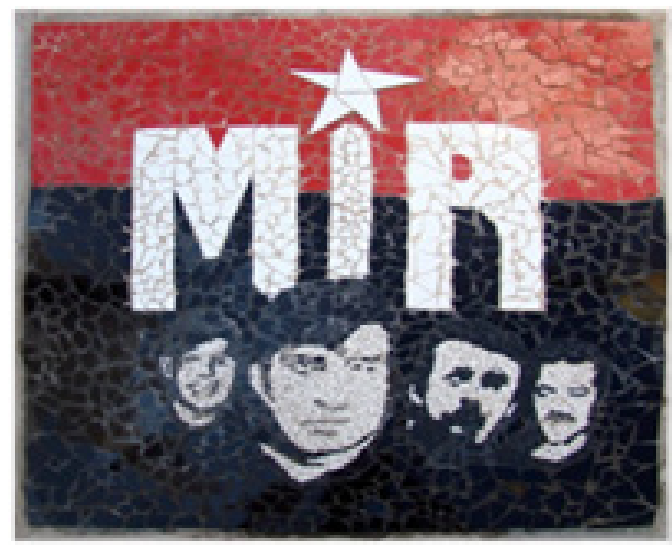

Por otro lado, los niños y niñas también desplegaban performances de recuerdo como el juego, la búsqueda y la imaginación. En la Imagen 3 podemos ver un grupo de niñas que recorre el Memorial buscando mosaicos. En estas búsquedas, las niñas corrían por el Memorial e inventaban historias sobre los mosaicos y las personas recordadas en ellos, acciones que destacaban por su carácter lúdico. El reconocimiento de estas prácticas nos muestra que hay otras formas de habitar el espacio y de participar en los procesos de memoria, propias de los niños y las niñas, ampliando así los significados y acciones con los cuales recordar.

\subsection{Lo que cuentan los niños y las niñas al hacer memoria}

Al usar el espacio para recordar, los niños y niñas que asisten al Memorial generan narraciones sobre el pasado, cuyo análisis nos permite entender qué y cómo recordamos (Vázquez, 2001). Si bien

10 Los nombres fueron reemplazados por pseudónimos para proteger la identidad de quienes participaron en la investigación. 
se ha pensado que las memorias de las nuevas generaciones son distintas a las de quienes vivieron los hechos recordados, y que el ejercicio de la transmisión es intrínsecamente transformador — y por ende sanador-, encontramos que gran parte de las memorias construidas por las niñas se relacionan justamente con las memorias que operan con mayor fuerza en nuestra sociedad: aquellas centradas en las víctimas y sus familiares como padecedores del daño y la desesperanza (Piper \& Montenegro, 2016).

Estas memorias se basan en la imposibilidad de acción, sobre lo cual decían las niñas que estas personas quedaban impotentes ante lo que les ocurría, fijando la memoria únicamente en la sensación de duelo. A su vez, el recuerdo del pasado desde esta dimensión invisibilizaba las historias de lucha social de los campesinos y campesinas de Paine durante el período de la reforma agraria, quedando la memoria anclada a un nivel individual y evitando el rescate del componente político de un sistema de violencia que ocurría a nivel social, lo que despolitizaba las memorias.

Sin embargo, también podemos ver que existen memorias transformadoras que son elaboradas desde afectos movilizadores como la esperanza y la justicia, lo que releva aspectos que van más allá de la situación victimizante, como la lucha que emprendieron las mujeres familiares en la búsqueda de los sujetos detenidos y desaparecidos. A su vez, estas memorias permiten posicionar a las personas recordadas desde sus experiencias cotidianas de vida, de lucha y de cambio social, conformándose de esta manera memorias de la resistencia y memorias vivas. La existencia de estas distintas memorias puede ser vista en las siguientes dos interpretaciones de mosaicos:

\section{Imágenes 6 y 7. Interpretaciones colectivas de mosaicos}

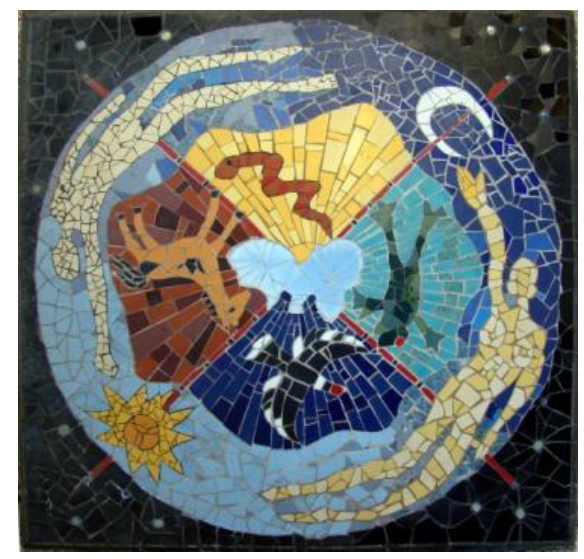

El negro es el terror que había afuera. Estos cristales son los sobrevivientes.

Hay una mujer y un hombre que se buscan pero nunca se encuentran.

Esto rojo es la sangre que corre por la dictadura.

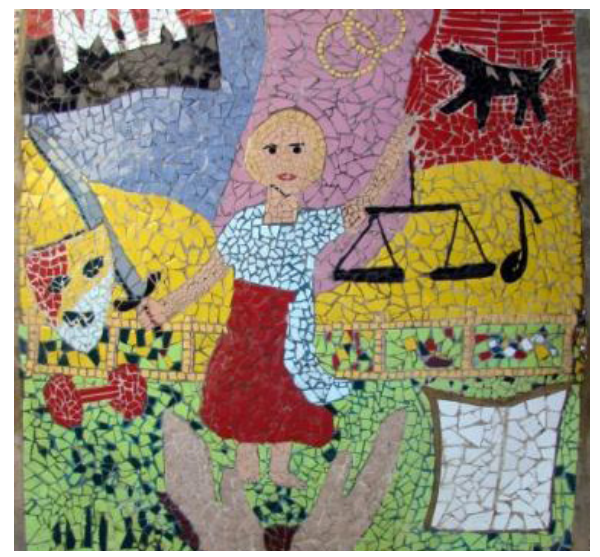

Ella está luchando por la justicia; esta balanza significa que debe haber igualdad.

El perdió a su perro.

Las pesas representan la fuerza de su lucha.

Se iba a casar. 
Como lo mostramos en los ejemplos, el uso de los mosaicos es central para la construcción de memoria, ya que su interpretación le da materialidad al recuerdo permitiendo una vinculación más cercana y tangible con el pasado. En sus interpretaciones, los niños y niñas hacían convivir historias biográficas, comunitarias y aspectos sociales del período de dictadura. Lo importante es que estas historias se elaboraban por medio de la imaginación, lo que posicionaba otras metáforas, significados y afectos.

De esta manera, el recuerdo se va configurando como un acto de creatividad que se realiza desde el presente, el cual toma elementos contingentes que se articulan en la imaginación de diversas historias (Fernández, 2000). Si bien la mezcla entre fantasía y memoria ha sido pensada como problemática porque alejaría a los niños y niñas de la verdad (Burman, 2008), lo que nos muestran las interpretaciones de mosaicos es que el carácter ficcional de su recuerdo es justamente el que releva la fuerza simbólica de la memoria (Piper et al., 2013), ya que posibilita la creación de discursos centrados en otros aspectos, ampliando la posibilidad de memorias que pueden ser construidas.

\section{Conclusiones}

En este artículo buscamos contribuir al análisis crítico de los procesos sociales en los que participa la infancia, procesos que nos dan la posibilidad de desnaturalizar las maneras en que entendemos a los niños y las niñas en nuestra sociedad, y a la vez nos permiten ampliar el campo de estudios de la memoria colectiva y de los derechos humanos al considerar la participación de la infancia en ellos.

Las memorias que construyen los niños y las niñas responden a un entramado complejo de significados y relaciones desplegados en el Memorial, excediendo muchas veces la relación de transmisión unilateral con los sujetos adultos. Desde esta óptica, el ejercicio de la memoria se realiza por medio de una co-construcción dialógica y participativa entre todos los actores, independientemente de su edad y experiencias. Esta construcción conjunta de memorias se encuentra atravesada por el tipo de relaciones y posiciones que van ocupando tanto niños y niñas como personas adultas, las cuales pueden ser más agenciales o pasivas. Los niños y las niñas son efectivamente partícipes relevantes cuando las memorias son hechas desde el presente, en acción cooperativa, y están fortalecidas por procesos de apropiación e identificación que consideran sus intereses en el espacio. Dentro de este contexto existen elementos fundamentales de apropiación e identificación, como las historias familiares propias o de otros sujetos, los aspectos de su cotidianidad representados en el espacio, y la vinculación de sus acciones y experiencias desde el presente. Por medio de estos elementos, los niños y niñas del Memorial Paine participan activamente del lugar y la memoria.

A través de su emergencia en el espacio, las memorias de las distintas generaciones se anudan en un mismo ejercicio de la memoria colectiva, en el que se ponen en tensión los diversos relatos, prácticas y relaciones por medio de las cuales se entraman las formas del recuerdo. Las memorias de los niños y niñas ponen en tensión los relatos autorizados del Memorial sobre el pasado, así como las pautas de comportamiento en el espacio a través de otras performances de memoria basadas en el juego y en la imaginación, aportando a su transformación por medio de la interacción con el. En ese sentido, las memorias de las distintas generaciones no debieran entenderse como esencialmente diferentes, sino como prácticas interdependientes y dinámicas que pueden cuestionar o reforzar un discurso oficial sobre el pasado en su interacción.

Al pensar que la transmisión intergeneracional del recuerdo tiene esencialmente una capacidad sanadora o transformadora, corremos el riesgo de idealizar un fenómeno más complejo que depende de los significados, de los contextos y de los actores que entran en juego al momento de recordar. Si bien el proyecto del Memorial se plantea abiertamente como una propuesta centrada en las historias de vida y en los proyectos políticos de las personas recordadas en el espacio, las construcciones de memoria en muchos de los Encuentros estuvieron más bien centradas en la muerte y en la represión durante la dictadura. En ese sentido, la construcción del pasado entre las distintas generaciones puede seguir reproduciendo memorias basadas en el daño, en la desesperanza y en la imposibilidad de acción. 
Lo que permite la transformación de esto último es un debate por medio de materialidades diversas, con aspectos basados en la resistencia, en la vida y en la esperanza. Tener esto en consideración entrega la posibilidad de desplazar visiones centradas en el deber de la transmisión, para dar paso a perspectivas que fomenten el sentirse parte de esta construcción y desde allí proponer memorias transformadoras que, a través de significados más agenciales, nos permitan pensar en mejores futuros posibles (Fernández, 2000).

Por otro lado, la memoria como un centro articulador de la vida social y de las prácticas políticas (Piper et al., 2017) tiene un fuerte potencial para relevar la agencia de los niños y niñas en los procesos sociales, no solo desde el pasado, sino también desde la acción en el presente y en el futuro. En ese sentido, consideramos los lugares de memoria como espacios abiertos para la politización de los discursos y acciones de las nuevas generaciones, que se constituyen en plataformas válidas para la participación ciudadana de la infancia. Por ello, el desafío al que buscamos contribuir por medio de esta publicación, es a la generación de prácticas de memoria participativas comprometidas con este fin, ya que así podremos promover, además de sociedades preocupadas de la memoria y de los derechos humanos, sociedades menos adultocéntricas y más abanderadas por la inclusión equitativa de las nuevas generaciones.

Finalmente, dentro del ámbito de estudio de infancia y memoria, en esta investigación abarcamos la observación de actividades concretas, logrando resultados contextuales e identificaciones particulares las cuales, si bien son ricas en contenido, no pueden ser extrapoladas a otras experiencias. De esta manera, para futuras líneas de investigación sería relevante la consideración de otros grupos de niños y niñas en distintos espacios de memoria, con el fin de explorar otras experiencias intergeneracionales en las que confluyen nuevos elementos a tener en consideración.

\section{Lista de referencias}

Alfageme, E., Cantos, R., \& Martínez, M. (2003). De la participación al protagonismo infantil: propuestas para la acción. Madrid: Editorial Plataforma de Organizaciones de Infancia.

Álvarez-Martínez-Conde, C., \& Hedrera, L. (2013). Puente Bulnes como lugar de memoria: tipos de apropiación y generación del Memorial. Quaderns de Psicología, 15(2), 55-62.

Batallán, G., \& Campanini, S. (2008). La participación política de niñ@s y jóvenes-adolescentes. Contribución al debate sobre la democratización de la escuela. Cuadernos de Antropología Social, (28), 85-106.

Burman, E. (2007). ¿Feminismo(s) o feminización? Entre el triunfalismo autónomo y la victimización. En Círculo de Bellas Artes (Comp.), La fragilización de las relaciones sociales. (pp. 109-130). Madrid: Círculo de Bellas Artes.

Burman, E. (2008). The child, the woman and the cybor: (Im)possibilities of feminist developmental psychology. En E. Burman (2008), Developments: Child, image, nation. (pp. 40-55). Nueva York: Routledge.

Burman, E. (2013). Entre dos deudas: niño y desarrollo (inter)nacional. Teoría y Crítica de la Psicología, 3, 3-19.

Burman, E., \& Stacey, J. (2010). The child and the childhood in feminist theory. Feminist Theory, 11(3), 227-240. doi:10.1177/1464700110376288

Bustelo, E. (2005). Infancia en indefensión. Salud Colectiva, 1(3), 253-284. doi:10.18294/sc.2005.47

Castillo-Gallardo, P. E., \& González-Celis, A. (2015). Infancia, dictadura y resistencia: hijos e hijas de la izquierda chilena (1973-1989). Revista Latinoamericana de Ciencias Sociales, Niñez y Juventud, 13(2), 907-921. doi:10.11600/1692715x.13225290914

Comisión Nacional de Reparación y Reconciliación. (1996). Informe de la Comisión Nacional de Verdad y Reconciliación (Informe Rettig). Santiago de Chile: Gobierno de Chile.

Corporación Opción. (2013). Los ruidos del silencio: los niños, niñas y adolescentes hablan a cuarenta años del golpe militar en Chile. Santiago de Chile: LOM Editores. 
Cruz, M.A., Reyes, M. J., \& Cornejo, M. (2012). Conocimiento situado y el problema de la subjetividad del investigador/a. Cinta de Moebio, 45, 253-274. doi:10.4067/S0717-554X2012000300005

Delgadillo, J. M. S. (2016). ¿Prevenir o reaccionar?, ¿consensuar o imponer? Estilos y costos políticos de las políticas de la memoria en Argentina y Chile. En G. Mira, \& F. Pedrosa (Eds.), Extendiendo los límites: nuevas agendas en historia reciente. (pp. 325-264). Salamanca: Ediciones Universidad de Salamanca.

Fernández, P. (2000). La afectividad colectiva. México, D. F.: Taurus.

Guber, R. (2011). La etnografia: método, campo y reflexividad. Buenos Aires: Siglo XXI.

Habashi, J. (2013). Palestinian children: Authors of collective memory. Children \& Society, 27, 421433. doi:10.1080/14733285.2011.562377

Halbwachs, M. (1968). La memoria colectiva. Zaragoza: Prensas Universitarias de Zaragoza.

Haraway, D. (1995). Ciencia, cyborgs y mujeres: la reinvención de la naturaleza. Madrid: Cátedra.

Haudrup, P. (2004). Children's participation in ethnographic research: Issues of power and representation. Children \& Society, 18, 165-176. doi:10.1002/CHI.823

Hite, K. (2013). Politica y arte de la conmemoración: memoriales en América Latina y España. Santiago de Chile: La Mandrágora.

Íñiguez, L. (2005). Nuevos debates, nuevas ideas y nuevas prácticas en la psicología social de la era 'post-construccionista'. Athenea Digital, 8. 1-7. doi:http://dx.doi.org/10.5565/rev/athenead/ v1n8.235

James, A., \& Prout, A. (2003). Constructing and reconstructing childhood: Contemporary issues in the sociological study of childhood. Londres: Taylor \& Francis.

Jelin, E. (2002). Los trabajos de la memoria. Madrid: Siglo XXI.

Kaufman, S. (2006). Lo legado y lo propio: lazos familiares y transmisión de memorias. En E. Jelin, \& S. Kaufman (Comps.), Subjetividad y figuras de la memoria. (pp. 47-71). Buenos Aires: Siglo XXI.

Leccardi, C., \& Feixa, C. (2011). El concepto de generación en las teorías sobre la juventud. Última Década, (34), 11-32.

Maillard, C., \& Ochoa, G. (2014). Yo soy... Mujeres familiares de detenidos desaparecidos y ejecutados de Paine. Santiago de Chile: Germina.

Mannion, G. (2007). Going spatial, going relational: Why 'listening to children' and children's participation needs reframing. Discourse, 28(3), 405-420.

Maureira, J. R. (2008). Enfrentar con la vida a la muerte: historias y memorias de la violencia y el terrorismo de estado en Paine (1960-2008). (Tesis de Licenciatura). Universidad de Chile, Santiago de Chile, Chile.

Memorial Paine. (2013). Plan de Gestión para Un lugar para la memoria Paine. Documento interno del Proyecto para Coalición Internacional de Sitios de Conciencia. Santiago de Chile: Memorial Paine.

Montenegro, M.(2001). Conocimientos, agentes y articulaciones: una mirada situada a la intervención social. (Tesis doctoral). Universidad Autónoma de Barcelona, Barcelona, España.

Piper, I. (2002). Sobre una práctica que, en el sur, se llama a sí misma psicología social crítica. En I. Piper (Comp.), Políticas, sujetos y resistencias: debates y críticas en psicología social. Santiago de Chile: Arcis.

Piper, I., Fernández, R., \& Íñiguez, L. (2013). Psicología social de la memoria: espacios y políticas del recuerdo. Psykhe, 22(2), 19-31. doi:10.7764/psykhe.22.2.574

Piper, I., \& Hevia, E. (2012). Espacio y recuerdo: archipiélago de memorias en Santiago de Chile. Santiago de Chile: Ocho Libros.

Piper, I., \& Montenegro, M. (2016). Ni víctimas, ni héroes, ni arrepentido/as. Reflexiones en torno a la categoría «víctima» desde el activismo político. Revista de Estudios Sociales, 59, 98-109. doi:https://dx.doi.org/10.7440/res59.2017.08 
Piper, I., Montenegro, M., Fernández, R., \& Sepúlveda, M. (2017). Places of memory. Visiting experiences in Santiago de Chile. Memory Studies. doi:10.1177/1750698017693667

Piper, I., Reyes, M. J., Fernández, R., Hevia, E., Badilla, M., \& Olivari, A. (2011). Lugares de memoria en Santiago de Chile: análisis visual de la construcción de sujetos. En C. Cisneros (Ed.), Análisis cualitativo asistido por computadora: teoría e investigación, (pp. 209-233). México, D. F.: Universidad Autónoma Metropolitana.

Programa de Derechos Humanos. (2013). Memoriales/Que son. Recuperado de: http://www.ddhh. gov.cl/memoriales.html

Restrepo, E. (2015). El proceso de investigación etnográfica: consideraciones éticas. Etnografias Contemporáneas, 1(1), 162-179.

Reyes, M. J. (2009). Generaciones de memoria: una dialógica conflictiva. Revista Praxis 10(15): 7797.

Reyes, M. J., Cornejo, M., Cruz, M. A., Carrillo, C., \& Caviedes, P. (2014). Dialogía intergeneracional en la construcción de memorias acerca de la dictadura militar chilena. Universitas Psychologica, 14(1), 255-270. doi:http://dx.doi. org/10.11144/Javeriana.upsy13-5.dicm

Reyes, M. J., Muñoz, J., \& Vázquez, F. (2013). Políticas de memoria desde los discursos cotidianos: la despolitización del pasado reciente en el Chile actual. Psykhe, 22(2), 161-173. doi:10.7764/ psykhe.22.2.582

Salomone, A., \& Cea, K. (2013). Memoria poética e infancia en la escritura de Antonia Torres y Alejandra del Río. Aisthesis, (54), 353-369.

Sarlo, B. (2005). Tiempo pasado: cultura de la memoria y giro subjetivo: una discusión. Buenos Aires: Siglo XXI.

Scott, J. (2001). Experiencia. La Ventana, (13), 42-73.

Vázquez, F. (2001). La memoria como acción social. Barcelona: Paidós

Veneros, D., \& Toledo, M. (2009). Del uso pedagógico de lugares de memoria: visita de estudiantes de educación media al parque por la paz Villa Grimaldi (Santiago, Chile). Estudios Pedagógicos, 35(1), 199-220.

Vergara, A. (2009). La intervención social como conflicto: el caso de la infancia y juventud en Chile. El Observador, (3), 19-35.

Vergara, A., Chávez, P., Peña, M., \& Vergara, E. (2016). Experiencias contradictorias y demandantes: la infancia y la adultez en la perspectiva de niños y niñas de Santiago de Chile. Revista Latinoamericana de Ciencias Sociales, Niñez y Juventud, 14(2), 1235-1247. doi:10.11600/1692 $715 x .14224051115$

Vergara, A., Peña, M., Chávez, P., \& Vergara, E. (2015). Los niños como sujetos sociales: el aporte de los nuevos estudios sociales de la infancia y el análisis crítico del discurso. Psicoperspectivas, 14(1), 55-65. doi:10.5027/PSICOPERSPECTIVAS-VOL14- ISSUE1- FULLTEXT-544.

Viú, D., \& Buzaglo, A. (2006). No hay punto final: los derechos humanos y las marcas en el espacio público. Recuperado de: http://www.viubuzaglo.com.ar/publicaciones/No_hay_punto_final.pdf 